\title{
Saturation points on faces of a rational polyhedral cone
}

\author{
Akimichi Takemura and Ruriko Yoshida
}

\begin{abstract}
Different commutative semigroups may have a common saturation. We consider distinguishing semigroups with a common saturation based on their "sparsity". We propose to qualitatively describe sparsity of a semigroup by considering which faces of the corresponding rational polyhedral cone have saturation points. For a commutative semigroup we give a necessary and sufficient condition for determining which faces have saturation points. We also show that we can construct a commutative semigroup with arbitrary consistent patterns of faces with saturations points.
\end{abstract}

Key words: antichain, face poset, Hilbert basis, hole, monoid, semigroup.

\section{Introduction}

Let $A=\left\{\boldsymbol{a}_{1}, \ldots, \boldsymbol{a}_{n}\right\}, \boldsymbol{a}_{i} \in \mathbb{Z}^{d}, i=1, \ldots, n$, be a finite set of integral points and let $Q=Q(A)$ denote the commutative semigroup generated by $\boldsymbol{a}_{1}, \ldots, \boldsymbol{a}_{n}$. In our previous paper (Takemura and Yoshida [2006]) we studied properties of holes, which are the difference between the semigroup and its saturation. We gave some necessary and sufficient conditions for the finiteness of the set of holes. In this paper we give a more detailed description of how holes are located, when there are infinitely many holes. Studying holes of $Q$ finds applications in many areas, such as commutative algebra (Bruns et al. [1997], Bruns and Gubeladze [2002b, a], Kantor and Sarkaria 2001], Gelfand et al. 1994], Sturmfels [1996]), optimization (Aardal and Lenstra 2002], Aardal et al. 2002, 2000]), number theory (Barvinok and Woods 2003|), and statistics (Dobra et al. [2003], Cox 2000, 2002], Ohsugi and Hibi [2006], Vlach [1986]).

Let $K=$ cone $\left(\boldsymbol{a}_{1}, \ldots, \boldsymbol{a}_{n}\right)$ be the rational polyhedral cone generated by $\boldsymbol{a}_{1}, \ldots, \boldsymbol{a}_{n}$. In this paper, without essential loss of generality, we assume that the lattice generated by $\boldsymbol{a}_{1}, \ldots, \boldsymbol{a}_{n}$ is $\mathbb{Z}^{d}$. In this case the saturation $Q_{\text {sat }}$ of $Q$ is defined by $Q_{\text {sat }}=K \cap \mathbb{Z}^{d}$. The elements of $H=Q_{\text {sat }} \backslash Q$ are called holes of $Q$. We assume that $K$ is a pointed cone with non-empty interior. Let $B$ denote the unique minimal Hilbert basis of $K$ (i.e. the unique minimal generator of $K \cap \mathbb{Z}^{d}$ ). In the following we simply say the Hilbert basis instead of the unique minimal Hilbert basis.

All holes have to be close to the boundary of $K$. However holes may be concentrated only around some low dimensional proper faces of $K$ or they may be distributed all over the boundary of $K$. In the latter case $Q$ can be considered as more sparse than the former case. In order to qualitatively describe this distinction 
we define the notion of an almost saturated face of $K$ and show how to determine whether a given face $F$ is almost saturated or not. In this paper we assume that $K$ is a given rational polyhedral cone and the purpose of our investigation is to differentiate semigroups with common saturation $K \cap \mathbb{Z}^{d}$ by their configurations of the almost saturated faces.

For the rest of this section we summarize some relevant definitions and results from Takemura and Yoshida 2006. We call $\boldsymbol{a} \in Q_{\text {sat }}, \boldsymbol{a} \neq 0$, a fundamental hole if $Q_{\text {sat }} \cap(\boldsymbol{a}+(-Q))=\{\boldsymbol{a}\}$. Let $H_{0}$ be the set of all fundamental holes in $Q$. $H_{0}$ is always finite for any given semigroup by Proposition 3.1 in Takemura and Yoshida [2006]. $\boldsymbol{a} \in Q$ is called a saturation point if $\boldsymbol{a}+Q_{\text {sat }} \subset Q$. Let $S$ be the set of all saturation points of the semigroup $Q$. Under the assumption that $K$ is pointed, $S$ is non-empty by Problem 7.15 of Miller and Sturmfels [2005]. Let $\bar{S}=Q \backslash S=$ non-saturation points of $Q$.

Now, consider minimal points of $S$ with respect to $S$ or $Q$. We call $\boldsymbol{a} \in S$ an $S$-minimal (or a $Q$-minimal) saturation point if there exists no other $\boldsymbol{b} \in S, \boldsymbol{b} \neq \boldsymbol{a}$, such that $\boldsymbol{a}-\boldsymbol{b} \in S$ (or $Q$ ). Let $\min (S ; S)$ be the set of $S$-minimal saturation points and $\min (S ; Q)$ be the set of $Q$-minimal saturation points.

The following is a list of some notation:

$$
\begin{aligned}
Q & =\left\{\lambda_{1} \boldsymbol{a}_{1}+\cdots+\lambda_{n} \boldsymbol{a}_{n}: \lambda_{1}, \cdots, \lambda_{n} \in \mathbb{N}=\{0,1, \ldots\}\right\} \\
K & =\left\{\lambda_{1} \boldsymbol{a}_{1}+\cdots+\lambda_{n} \boldsymbol{a}_{n}: \lambda_{1}, \cdots, \lambda_{n} \in \mathbb{R}_{+}\right\} \\
Q_{\text {sat }} & =K \cap \mathbb{Z}^{d}=\text { saturation of } A \supset Q \\
H & =Q_{\text {sat }} \backslash Q=\text { holes in } Q_{\text {sat }} \\
H_{0} & =\left\{\boldsymbol{a} \in Q_{\text {sat }}: Q_{\text {sat }} \cap(\boldsymbol{a}+(-Q))=\{\boldsymbol{a}\}, \boldsymbol{a} \neq 0\right\} \\
& =\text { fundamental holes in } Q_{\text {sat }} \\
S & =\left\{\boldsymbol{a} \in Q: \boldsymbol{a}+Q_{\text {sat }} \subset Q\right\}=\text { saturation points of } Q \\
\bar{S} & =Q \backslash S=\text { non-saturation points of } Q \\
\min (S ; S) & =\{\boldsymbol{a} \in S:(\boldsymbol{a}+(-(S \cup\{0\}))) \cap S=\{\boldsymbol{a}\}\} \\
& =\text { minimal } S \text {-saturation points of } Q \\
\min (S ; Q) & =\{\boldsymbol{a} \in S:(\boldsymbol{a}+(-Q)) \cap S=\{\boldsymbol{a}\}\} \\
& =\text { minimal } Q \text {-saturation points of } Q
\end{aligned}
$$

Using these definitions and notation, Takemura and Yoshida 2006] showed several necessary and sufficient conditions for $H$ 's finiteness, which are summarized in the following theorem:

Theorem 1.1 (Takemura and Yoshida 2006]). Under the assumptions and the definitions above the following statements are equivalent:

(1) $\min (S ; S)$ is finite.

(2) cone $(S)$ is a closed rational polyhedral cone.

(3) There is some $s \in S$ on every extreme ray of $K$.

(4) $\mathrm{H}$ is finite.

(5) $\bar{S}$ is finite.

In this paper, we further investigate saturation points and holes in a semigroup with the given polyhedral cone $K$ and we study how saturation points are distributed in each face of the fixed polyhedral cone $K$. In Section 2, we will define 
almost saturated faces and nowhere saturated faces of $K$. Then, we will extend the results in Takemura and Yoshida [2006] in terms of almost saturated faces and nowhere saturated faces of $K$, and we will show some preliminary results on almost saturated faces and nowhere saturated faces of $K$. In Section 3 , we will give the necessary and sufficient conditions for a face of $K$ to be almost saturated or to be nowhere saturated. The results in Section 4 show that one can construct a semigroup $Q$ from any antichain of faces of any given cone $K$ so that the faces in the antichain are minimal almost saturated in $Q$. In Section [5 we will apply our theorems to a small example and to a more complicated example of $2 \times 2 \times 2 \times 2$ tables with three 2 -marginals and a 3 -marginal as the simplicial complex on 4 nodes [12][13][14][234] with levels of 2 on each node.

\section{Definitions and preliminary results}

Let $\mathcal{F}$ denote the face poset of $K$. For each proper face $F \in \mathcal{F}, F \subsetneq K$, there exists a supporting hyperplane

$$
L_{F}=\left\{\boldsymbol{u} \in \mathbb{R}^{d} \mid \boldsymbol{c}_{F} \cdot \boldsymbol{u}=0\right\}
$$

such that $\boldsymbol{c}_{F} \cdot \boldsymbol{u} \geq 0, \forall \boldsymbol{u} \in K$, and

$$
F=L_{F} \cap K
$$

For each proper face $F$, we choose and fix $\boldsymbol{c}_{F}$ throughout this paper. $\operatorname{relint}(F)$ denotes the relative interior of $F$.

Let $A_{F}=A \cap F$ denote the set of elements of $A$ in the face $F$. Similarly define $Q_{F}=Q(A) \cap F$. Then

$$
Q_{F}=\operatorname{semigroup}\left(A_{F}\right)
$$

is the commutative semigroup generated by $\boldsymbol{a} \in A_{F}$. As a particular convenient element in $Q(A) \cap \operatorname{relint}(F)$, we often make use of the following element

$$
\boldsymbol{a}_{F}^{*}=\sum_{\boldsymbol{a}_{i} \in A_{F}} \boldsymbol{a}_{i} .
$$

Elements of the Hilbert basis $B$ in $F$ is denoted by

$$
B_{F}=B \cap F .
$$

$B_{F}$ is the unique minimal Hilbert basis for $F$. It can be easily shown that every element in the Hilbert basis $B$ not belonging to $Q$ is a fundamental hole:

$$
B \backslash Q \subset H_{0} \text {. }
$$

Now we give a key definition for this paper.

Definition 2.1. We call a face $F$ almost saturated if there exists a saturation point of $Q=Q(A)$ on $F$. Otherwise (i.e. if no point of $F$ is a saturation point) we call $F$ nowhere saturated.

For the one-dimensional faces (i.e. extreme rays) of $K$ this definition corresponds to Condition 3 of Theorem [1.1. The basic fact on the existence of saturation point (Problem 7.15 of Miller and Sturmfels 2005]) is that $K$ itself is always almost saturated. However generally we do not know whether any other face of $K$ is almost saturated or not. Therefore the important question is to ask which faces of $K$ are almost saturated if $H$ is infinite.

Now from the definition the following lemma is obvious. 
Lemma 2.2. Let $F, G$ be two faces of $K$ with $G \subset F$. If $G$ is almost saturated, then $F$ is almost saturated. Alternatively, if $F$ is nowhere saturated, then $G$ is nowhere saturated.

This lemma shows that if there are infinitely many holes, we can describe how the holes are distributed in terms of the set of minimal almost saturated faces or the set of maximal nowhere saturated faces. Here "minimal" and "maximal" refer to the partial order of the face poset in terms of inclusion of faces.

In the above lemma suppose that $\boldsymbol{b} \in G$ is a saturation point. Then for any $\boldsymbol{a} \in \operatorname{relint}(F), \boldsymbol{b}+\boldsymbol{a} \in \operatorname{relint}(F)$ and $\boldsymbol{b}+\boldsymbol{a}$ is a saturation point. Therefore if $F$ is almost saturated, then there always exists a saturation point in $\operatorname{relint}(F)$. Alternatively, $F$ is nowhere saturated if no point of $\operatorname{relint}(F)$ is a saturation point. We can summarize this fact as follows.

$$
F \cap S=\emptyset \quad \Leftrightarrow \quad \operatorname{relint}(F) \cap S=\emptyset .
$$

We end this section with some 2-dimensional examples to illustrate our definitions. The commutative semigroups of the examples share a common saturation but the distribution of the holes are different. In our examples we will write $A$ as a $d \times n$ integral matrix, so that $\boldsymbol{a}_{1}, \ldots, \boldsymbol{a}_{n}$ are columns vectors of $A$.

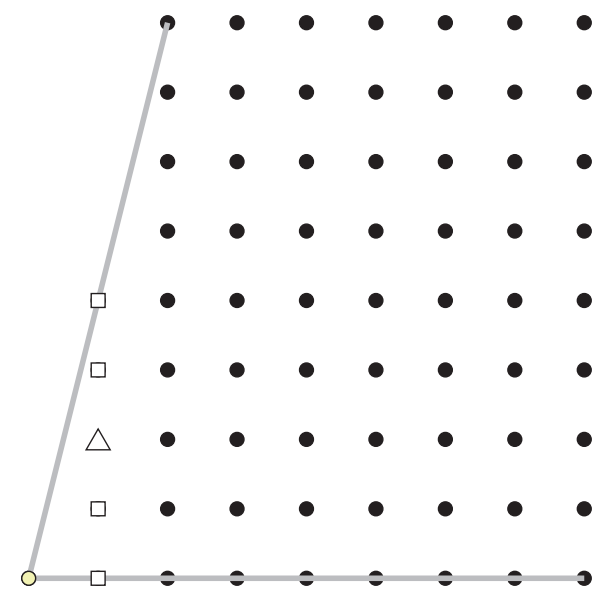

FiguRE 1. White circles represent nonsaturation points, a triangle represents a hole, white squares represent $S$-minimal saturation points, and black circles represent non $S$-minimal saturation points in the semigroup in Example 2.3.

Example 2.3. Let $A$ be an integral matrix such that

$$
A=\left(\begin{array}{llll}
1 & 1 & 1 & 1 \\
0 & 1 & 3 & 4
\end{array}\right) .
$$

The cone $K$ is defined by $K=\operatorname{cone}(A)=\left\{\left(x_{1}, x_{2}\right)^{t}:-4 x_{1}+x_{2} \leq 0, x_{1}, x_{2} \in\right.$ $\left.\mathbb{R}_{+}\right\}$. The set of holes $H$ consists of only one element $\left\{(1,2)^{t}\right\} . \bar{S}=\left\{(0,0)^{t}\right\}$. $\min (S ; S)=\left\{(1,0)^{t},(1,1)^{t},(1,3)^{t},(1,4)^{t}\right\}$ Thus, $H, \bar{S}$, and $\min (S ; S)$ are all finite. Note that one-dimensional faces $F_{1}=\left\{\left(x_{1}, 0\right)^{t}: x_{1} \in \mathbb{R}_{+}\right\}$and $F_{2}=$ $\left\{\left(x_{1}, x_{2}\right)^{t}:-4 x_{1}+x_{2}=0, x_{1}, x_{2} \in \mathbb{R}_{+}\right\}$contain saturation points in each relative interior (i.e. a saturation point $(2,0)^{t} \in \operatorname{relint}\left(F_{1}\right)$ and a saturation point 


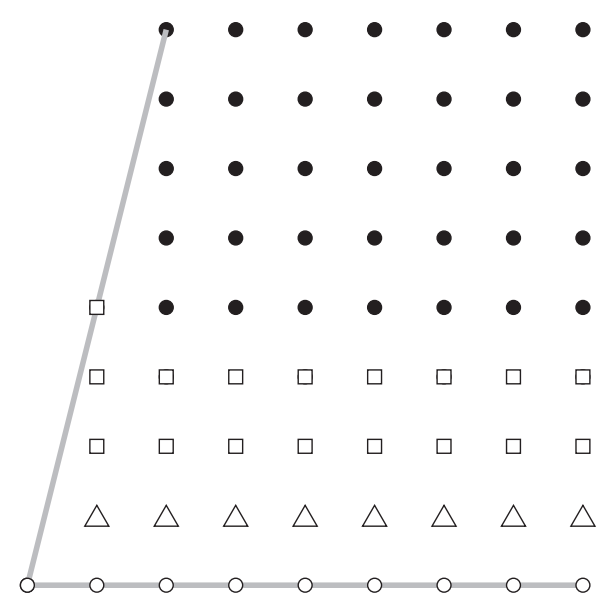

Figure 2. White circles represent nonsaturation points, triangles represent holes, white squares represent $S$-minimal saturation points, and black circles represent non $S$-minimal saturation points in the semigroup in Example 2.4.

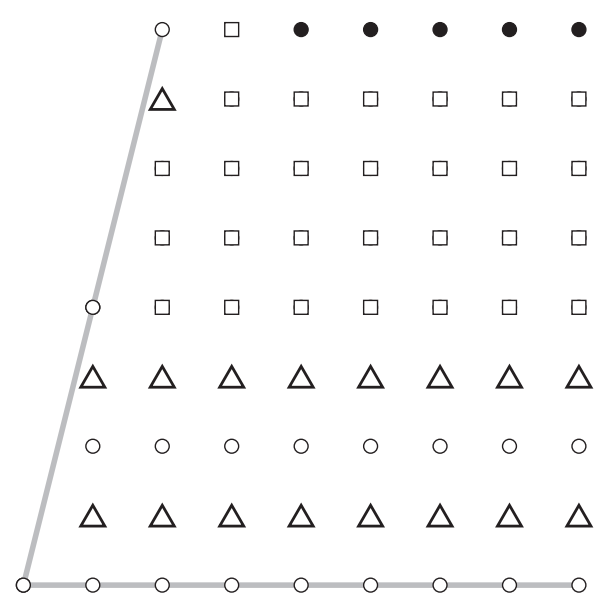

FiguRE 3. White circles represent nonsaturation points, triangles represent holes, white squares represent $S$-minimal saturation points, and black circles represent non $S$-minimal saturation points in the semigroup in Example 2.5.

$\left.(1,4)^{t} \in \operatorname{relint}\left(F_{2}\right)\right)$. Since $F_{1} \subset K$, by Lemma 2.2, $K$ is almost saturated and since $K$ contains saturation points $(2,0)^{t}$ and $(1,4)^{t}, K$ is indeed almost saturated.

Example 2.4. Let $A$ be an integral matrix such that

$$
A=\left(\begin{array}{cccc}
1 & 1 & 1 & 1 \\
0 & 2 & 3 & 4
\end{array}\right) .
$$

The cone $K$ is defined by $K=\operatorname{cone}(A)=\left\{\left(x_{1}, x_{2}\right)^{t}:-4 x_{1}+x_{2} \leq 0, x_{1}, x_{2} \in \mathbb{R}_{+}\right\}$ which is the same as Example 2.3. The set of holes $H$ consists of elements $\{(k, 1)$ : 
$k \in \mathbb{Z}, k \geq 1\} . \bar{S}=\left\{(i, 0)^{t}: i \in \mathbb{Z}, i \geq 0\right\}$. and $\min (S ; S)=\left\{(k, j)^{t}: k \in \mathbb{Z}, k \geq\right.$ $1,2 \leq j \leq 3\} \cup\{(1,4)\}$. Thus, $H, \bar{S}$, and $\min (S ; S)$ are all infinite. However, $\min (S ; Q)=\left\{(1,2)^{t},(1,3)^{t},(1,4)^{t}\right\}$ is finite. Note that a one-dimensional face $F_{2}=\left\{\left(x_{1}, x_{2}\right)^{t}:-4 x_{1}+x_{2}=0, x_{1}, x_{2} \in \mathbb{R}_{+}\right\}$contains a saturation point $(1,4)^{t} \in$ relint $\left(F_{2}\right)$ in its relative interior, so $F_{2}$ is almost saturated. However, another onedimensional face $F_{1}=\left\{\left(x_{1}, 0\right)^{t}: x_{1} \in \mathbb{R}_{+}\right\}$does not contains any saturation points in its relative interior, so $F_{1}$ is nowhere saturated. Since $\bar{S}=Q_{F_{1}}=Q \cap F_{1}$, $Q_{F_{1}}$ does not contain any saturation points and also note that since $F_{2}$ is almost saturated, $K$ is almost saturated ( $K$ contains a saturation point $(1,4)^{t}$ in its relative interior).

Example 2.5. Let $A$ be an integral matrix such that

$$
A=\left(\begin{array}{llll}
1 & 1 & 2 & 1 \\
0 & 2 & 5 & 4
\end{array}\right) .
$$

The cone $K$ is defined by $K=\operatorname{cone}(A)=\left\{\left(x_{1}, x_{2}\right)^{t}:-4 x_{1}+x_{2} \leq 0, x_{1}, x_{2} \in \mathbb{R}_{+}\right\}$ which is the same as Example 2.3 and Example 2.4. The set of holes $H$ consists of elements $\left\{(k, 1)^{t}: k \in \mathbb{Z}, k \geq 1\right\} \cup\left\{(k, 4 k-1)^{t}: k \in \mathbb{Z}, k \geq 1\right\} \cup\left\{(k, 3)^{t}: k \in \mathbb{Z}, k \geq\right.$ $1\} . \bar{S}=\left\{(i, 0)^{t}: i \in \mathbb{Z}, i \geq 0\right\} \cup\left\{(i, 2)^{t}: i \in \mathbb{Z}, i \geq 1\right\} \cup\left\{(i, 4 i)^{t}: i \in \mathbb{Z}, i \geq 0\right\}$ and $\min (S ; S)=\left\{(k, j)^{t}: k \in \mathbb{Z}, k \geq 2,4 \leq j \leq 6\right\} \cup\left\{(3,8)^{t}\right\} \cup\left\{(k, j)^{t}: k \in \mathbb{Z}, k \geq\right.$ $3, j=7\} \cup\left\{(k, 4 k-i)^{t}: k, i \in \mathbb{Z}, k \geq 3, i=2,3\right\}$. Thus, $H, \bar{S}$, and $\min (S ; S)$ are all infinite. However, $\min (S ; Q)=\left\{(2,4)^{t},(2,5)^{t},(2,6)^{t}\right\}$ is finite. Note that one dimensional faces $F_{1}=\left\{\left(x_{1}, 0\right)^{t}: x_{1} \in \mathbb{R}_{+}\right\}$and $F_{2}=\left\{\left(x_{1}, x_{2}\right)^{t}:-4 x_{1}+x_{2}=\right.$ $\left.0, x_{1}, x_{2} \in \mathbb{R}_{+}\right\}$do not contain any saturation points in its relative interior, so $F_{1}$ and $F_{2}$ are nowhere saturated. However, since $K$ contains a saturation point $(2,4)^{t}$, so $K$ is almost saturated.

\section{Condition for almost saturation of a face}

In this section we consider the problem of determining which face of $K=$ $\operatorname{cone}(A)$ is almost saturated for a given $A$. We use the following fact proved in Lemma 4.1 of Takemura and Yoshida [2006].

Lemma 3.1. Suppose that the semigroup $Q=Q(A)$ is not saturated. $\boldsymbol{a} \in Q$ is a saturation point if and only if $\boldsymbol{a}+\boldsymbol{y} \in Q$ for all fundamental holes $\boldsymbol{y}$.

For a face $F$, we denote the parallel shift of $Q_{F}$ by $\boldsymbol{y} \in K$ by

$$
\boldsymbol{y}+Q_{F}=\{\boldsymbol{y}+\boldsymbol{a} \mid \boldsymbol{a} \in Q \cap F\} .
$$

We will consider the case that $\boldsymbol{y}$ is a hole. We now prove the following fact.

Proposition 3.2. A face $F$ is nowhere saturated if and only if there exists some fundamental hole $\boldsymbol{y} \in H_{0}$ such that all points of $\boldsymbol{y}+Q_{F}$ are holes, i.e. $\boldsymbol{y}+Q_{F} \subset H$.

Proof. Suppose that $\boldsymbol{y}+Q_{F} \subset H$ for some fundamental hole $\boldsymbol{y} \in H_{0}$. Then for $\boldsymbol{y}+\boldsymbol{a} \notin Q$ for every $\boldsymbol{a} \in Q_{F}$. By Lemma 3.1 $\boldsymbol{a}$ is not a saturation point. Therefore $F$ is nowhere saturated.

Conversely suppose that $F$ is nowhere saturated. Then no $\boldsymbol{a} \in Q_{F}$ is a saturation point. By Lemma 3.1 for each $\boldsymbol{a} \in Q_{F}$ there exists a fundamental hole $\boldsymbol{y} \in H_{0}$ such that $\boldsymbol{a}+\boldsymbol{y} \in H$. Consider $\boldsymbol{a}_{F}^{*}$ in (1). $\boldsymbol{a}_{F}^{*} \in \operatorname{relint}(F) \cap Q$. Consider $\boldsymbol{a}_{F}^{*}, 2 \boldsymbol{a}_{F}^{*}, 3 \boldsymbol{a}_{F}^{*}, \ldots$ These are not saturation points of $Q$. Therefore for each $k \boldsymbol{a}_{F}^{*}$, $k \in \mathbb{N}$, there exists a fundamental hole $\boldsymbol{y}=\boldsymbol{y}_{k}$ such that $k \boldsymbol{a}_{F}^{*}+\boldsymbol{y} \in H$. Since 
there are only finite number of fundamental holes, there exists a fundamental hole $\boldsymbol{y}_{0} \in H_{0}$ such that $k \boldsymbol{a}_{F}^{*}+\boldsymbol{y}_{0} \in H$ for infinitely many $k$.

Now each $\boldsymbol{a} \in Q_{F}$ is a non-negative integer combination of $\boldsymbol{a}_{i}$ 's in $Q_{F}$ :

$$
\boldsymbol{a}=\sum_{\boldsymbol{a}_{i} \in A_{F}} x_{i} \boldsymbol{a}_{i}, \quad x_{i} \in \mathbb{N} .
$$

Choose $m \geq \max \left\{x_{i} \mid \boldsymbol{a}_{i} \in A_{F}\right\}$ such that $m \boldsymbol{a}_{F}^{*}+\boldsymbol{y}_{0} \in H$. Then

$$
m \boldsymbol{a}_{F}^{*}-\boldsymbol{a}=\sum_{\boldsymbol{a}_{i} \in A_{F}}\left(m-x_{i}\right) \boldsymbol{a}_{i} \in Q_{F} .
$$

Now if $\boldsymbol{a}+\boldsymbol{y}_{0} \in Q$, then

$$
m \boldsymbol{a}_{F}^{*}+\boldsymbol{y}_{0}=\boldsymbol{a}+\boldsymbol{y}_{0}+\left(m \boldsymbol{a}_{F}^{*}-\boldsymbol{a}\right) \in Q
$$

which is a contradiction. Therefore $\boldsymbol{a}+\boldsymbol{y}_{0} \in H$ for every $\boldsymbol{a} \in Q_{F}$.

We now state the following main result of this section.

Theorem 3.3. A face $F$ is almost saturated if and only if every fundamental hole $\boldsymbol{y} \in H_{0}$ can be written as

$$
\boldsymbol{y}=x_{1} \boldsymbol{a}_{1}+\cdots+x_{n} \boldsymbol{a}_{n}, \quad x_{j} \in \mathbb{Z}, \forall j, \quad \text { and } x_{j} \geq 0 \text { for } \boldsymbol{a}_{j} \notin F .
$$

Equivalently, $F$ is nowhere saturated if and only if (3) does not have a feasible solution for some fundamental hole $\boldsymbol{y}$.

Proof. Suppose that $F$ is almost saturated. Then by Proposition $3.2, \boldsymbol{y}+$ $Q_{F} \not \subset H$ for every fundamental hole $\boldsymbol{y}$. Therefore for every $\boldsymbol{y}$ there exists $\boldsymbol{a} \in Q_{F}$ such that $\boldsymbol{y}+\boldsymbol{a} \in Q$, i.e. $\boldsymbol{b}=\boldsymbol{y}+\boldsymbol{a} \in Q$. But then $\boldsymbol{y}=\boldsymbol{b}-\boldsymbol{a}$ is a feasible solution to (3). Therefore, every fundamental hole $\boldsymbol{y}$ can be written in the form in (3).

Conversely suppose that (31) has a feasible solution for every fundamental hole $\boldsymbol{y}$. Then

$$
\boldsymbol{y}+\sum_{\boldsymbol{a}_{i} \in F}\left|x_{i}\right| \boldsymbol{a}_{i}=\sum_{\boldsymbol{a}_{i} \in F}\left(x_{i}+\left|x_{i}\right|\right) \boldsymbol{a}_{i}+\sum_{\boldsymbol{a}_{i} \notin F} x_{i} \boldsymbol{a}_{i} \in Q .
$$

Since $\sum_{\boldsymbol{a}_{i} \in F}\left|x_{i}\right| \boldsymbol{a}_{i} \in Q_{F}$ and since $\boldsymbol{y}+\sum_{\boldsymbol{a}_{i} \in F}\left|x_{i}\right| \boldsymbol{a}_{i} \in Q, F$ is almost saturated by Proposition 3.2 .

Theorem 3.3 is stated in terms of the fundamental holes. For applications it is more convenient to have a condition for elements in the Hilbert basis $B$ of $K$. We have the following theorem.

Theorem 3.4. A face $F$ is nowhere saturated if and only if for some element $\boldsymbol{b}$ of the Hilbert basis $B$

$$
\boldsymbol{b}=x_{1} \boldsymbol{a}_{1}+\cdots+x_{n} \boldsymbol{a}_{n}, \quad x_{j} \in \mathbb{Z}, \forall j, \quad \text { and } x_{j} \geq 0 \text { for } \boldsymbol{a}_{j} \notin F .
$$

does not have a feasible solution.

Proof. Suppose that (4) does not have a feasible solution for some $\boldsymbol{b} \in B$. Obviously this $\boldsymbol{b}$ can not belong to $Q$. Then by (2) $\boldsymbol{b}$ is a fundamental hole and $F$ is nowhere saturated by Theorem 3.3 .

Conversely suppose that (4) has a feasible solution for every element $\boldsymbol{b} \in B$. Now every fundamental hole $\boldsymbol{y} \in H_{0}$ can be written as a non-negative integral combination of $\boldsymbol{b}$ 's. Then forming the same non-negative integral combination of the feasible solutions of $\boldsymbol{b}$ 's, we obtain a feasible solution to (3) for every fundamental hole $\boldsymbol{y} \in H_{0}$. Therefore by Theorem $3.3 F$ is almost saturated. 
Finally we prove the following theorem.

Theorem 3.5. A face $F$ is nowhere saturated if and only if there exists a $Q$ minimal saturation point $\boldsymbol{y}$ such that every point of $\boldsymbol{y}+Q_{F}$ is an S-minimal saturation point.

Proof. The set of $Q$-minimal saturation points $\min (S ; Q)$ is always finite by Proposition 4.4 of Takemura and Yoshida [2006]. Suppose that $F$ is nowhere saturated. Then $\min (S ; Q) \cap F=\emptyset$. In Section 1 , we chose the normal vector $\boldsymbol{c}_{F}$ for $F$. Choose a point $\boldsymbol{y}_{0}$ from $\min (S ; Q)$ which minimizes $\boldsymbol{c}_{F} \cdot \boldsymbol{y}$ :

$$
\boldsymbol{c}_{F} \cdot \boldsymbol{y}_{0}=\min _{\boldsymbol{y} \in \min (S ; Q)} \boldsymbol{c}_{F} \cdot \boldsymbol{y}>0 \text {. }
$$

Because $\boldsymbol{y}_{0}$ is a saturation point, all points on $\boldsymbol{y}_{0}+Q_{F}$ are saturation points. Suppose that some $\boldsymbol{a} \in \boldsymbol{y}_{0}+Q_{F}$ is not an $S$-minimal saturation point. Then $\boldsymbol{a}$ can be written as a sum of non-zero saturation points

$$
\boldsymbol{a}=\boldsymbol{a}_{1}+\boldsymbol{a}_{2}, \quad \boldsymbol{a}_{1}, \boldsymbol{a}_{2} \in S
$$

and $\boldsymbol{c}_{F} \cdot \boldsymbol{a}=\boldsymbol{c}_{F} \cdot \boldsymbol{a}_{1}+\boldsymbol{c}_{F} \cdot \boldsymbol{a}_{2}$. Since $\boldsymbol{a}_{1}, \boldsymbol{a}_{2}$ do not belong to $Q_{F}$, both $\boldsymbol{c}_{F} \cdot \boldsymbol{a}_{1}$ and $\boldsymbol{c}_{F} \cdot \boldsymbol{a}_{2}$ are positive. In particular $\boldsymbol{c}_{F} \cdot \boldsymbol{a}>\boldsymbol{c}_{F} \cdot \boldsymbol{a}_{1}>0$. However $\boldsymbol{c}_{F} \cdot \boldsymbol{a}=\boldsymbol{c}_{F} \cdot \boldsymbol{y}_{0}$ and

$$
\boldsymbol{c}_{F} \cdot \boldsymbol{y}_{0}>\boldsymbol{c}_{F} \cdot \boldsymbol{a}_{1}>0
$$

Now $\boldsymbol{a}_{1}$ can be written as $\boldsymbol{a}_{1}=\boldsymbol{y}_{1}+\tilde{\boldsymbol{y}}, \boldsymbol{y}_{1} \in \min (S ; Q), \tilde{\boldsymbol{y}} \in Q$, and therefore $\boldsymbol{c}_{F} \cdot \boldsymbol{a}_{1} \geq \boldsymbol{c}_{F} \cdot \boldsymbol{y}_{1}$. Then $\boldsymbol{c}_{F} \cdot \boldsymbol{y}_{0}>\boldsymbol{c}_{F} \cdot \boldsymbol{y}_{1}>0$, but this contradicts our choice of $\boldsymbol{y}_{0}$. Therefore $\boldsymbol{y}_{0}+Q_{F} \subset \min (S ; S)$.

Conversely suppose that $F$ is almost saturated. Then there exists a saturation point $\boldsymbol{a}$ on $Q_{F}$. Let $\boldsymbol{y}$ be any $Q$-minimal saturation point and consider $\boldsymbol{y}+\boldsymbol{a}$, which is a sum of two non-zero saturation points and therefore not $S$-minimal. But $\boldsymbol{y}+\boldsymbol{a} \in \boldsymbol{y}+Q_{F}$. Therefore $\boldsymbol{y}+Q_{F} \not \subset \min (S ; S)$.

\section{Construction of a semigroup with arbitrary configuration of almost saturated faces}

The set of minimal almost saturated faces form an antichain (Section 3.1 of Stanley [1997]), i.e. there is no inclusion relation among minimal almost saturated faces. Then a natural question to ask is whether for any given antichain of faces we can construct $Q=Q(A)$ such that the set of minimal almost saturated faces of $Q$ coincides with the given antichain. In this section we show that it is always possible by explicitly constructing $A$ for a given antichain.

Let $\left\{F_{1}, \ldots, F_{M}\right\}$ be an antichain of faces. Write

$$
\bar{B}=B \cup\{0\},
$$

which is the Hilbert basis with the origin added. For $F_{i}, i=1, \ldots, M$, let

$$
\boldsymbol{b}_{F_{i}}^{*}=\sum_{\boldsymbol{b} \in B_{F_{i}}} \boldsymbol{b} \in \operatorname{relint}\left(F_{i}\right) \text {. }
$$

Theorem 4.1. For any given antichain of faces $\left\{F_{1}, \ldots, F_{M}\right\}$ let

$$
A=\{2 \boldsymbol{b} \mid \boldsymbol{b} \in B\} \cup\left(\left\{2 \boldsymbol{b}_{F_{1}}^{*}, \ldots, 2 \boldsymbol{b}_{F_{M}}^{*}\right\}+\bar{B}\right),
$$

Then we have i) cone $(A)=K$, ii) the saturation of $Q(A)$ is $K \cap \mathbb{Z}^{d}$, iii) the minimal almost saturated faces for $Q(A)$ are $F_{1}, \ldots, F_{M}$. 
Note that each element of $A$ is either i) $2 \boldsymbol{b}, \boldsymbol{b} \in B$, ii) $2 \boldsymbol{b}_{F_{i}}^{*}, i=1, \ldots, M$, iii) or of the form $2 \boldsymbol{b}_{F_{i}}^{*}+\boldsymbol{b}, \boldsymbol{b} \in B$.

Proof. $\operatorname{cone}(A)=K$ because the Hilbert basis $B$ contains all the extreme rays of $K$ and $A$ contains all $2 \boldsymbol{b}, \boldsymbol{b} \in B$. The saturation of $Q(A)$ is $K \cap \mathbb{Z}^{d}$ because $K \cap \mathbb{Z}^{d}$ is generated by $B$ and each $\boldsymbol{b} \in B$ can be written as

$$
\boldsymbol{b}=\left(2 \boldsymbol{b}_{F_{1}}^{*}+\boldsymbol{b}\right)-2 \boldsymbol{b}_{F_{1}}^{*},
$$

where both $2 \boldsymbol{b}_{F_{1}}^{*}+\boldsymbol{b}$ and $2 \boldsymbol{b}_{F_{1}}^{*}$ belong to $A$.

Now we show that every face $G \in \mathcal{G}$ is nowhere saturated, where

$$
\mathcal{G}=\left\{G \mid G \not \supset F_{i}, \quad \forall i=1, \ldots, M\right\} .
$$

For an arbitrary $G \in \mathcal{G}$ consider $\boldsymbol{c}_{G}$ chosen in Section 2. $G$ is a proper face and there exists $\boldsymbol{b} \in B$ such that $\boldsymbol{b} \notin G$. For each $G$ choose $\boldsymbol{b}_{G} \in B \backslash G$ such that

$$
\boldsymbol{c}_{G} \cdot \boldsymbol{b}_{G}=\min _{\boldsymbol{b} \in B \backslash G} \boldsymbol{c}_{G} \cdot \boldsymbol{b}>0 .
$$

Now for an arbitrary $G \in \mathcal{G}$ consider elements $\boldsymbol{x}$ on $\boldsymbol{b}_{G}+Q_{G}$. Then $\boldsymbol{c}_{G} \cdot \boldsymbol{x}=\boldsymbol{c}_{G} \cdot \boldsymbol{b}_{G}$ for all $\boldsymbol{x} \in \boldsymbol{b}_{G}+Q_{G}$. On the other hand let $\boldsymbol{a} \in Q(A) \backslash G$. Since $\boldsymbol{a}$ is a non-negative integral combination of the elements of $A$, we distinguish two cases: i) $\boldsymbol{a}$ contains some $2 \boldsymbol{b}, \boldsymbol{b} \notin G$, or ii) $\boldsymbol{a}$ does not contain any $2 \boldsymbol{b}, \boldsymbol{b} \notin G$. In the former case we have

$$
\boldsymbol{c}_{G} \cdot \boldsymbol{a} \geq 2 \boldsymbol{c}_{G} \cdot \boldsymbol{b} \geq 2 \boldsymbol{c}_{G} \cdot \boldsymbol{b}_{G}>\boldsymbol{c}_{G} \cdot \boldsymbol{b}_{G}
$$

and $\boldsymbol{a} \notin \boldsymbol{b}_{G}+Q_{G}$. For the latter case $\boldsymbol{a}$ has to contain some $2 \boldsymbol{b}_{F_{i}}^{*}$. Furthermore, since $\boldsymbol{a}$ is not on $G$, this $F_{i}$ is not a subset of $G$ and therefore $\boldsymbol{b}_{F_{i}}^{*}$ contains some $\boldsymbol{b} \notin G$. Then the same argument as in the case i) shows that $\boldsymbol{a} \notin \boldsymbol{b}_{G}+Q_{G}$. We have shown that all points of $\boldsymbol{b}_{G}+Q_{G}$ are holes and hence $G$ is nowhere saturated by Proposition 3.2 .

Finally we need to show that each $F_{i}$ is almost saturated. Let $C=|B|$ denote the number of elements in the Hilbert basis. We claim that $2 C \boldsymbol{b}_{F_{i}}^{*}$ is a saturation point and therefore $F_{i}$ is almost saturated. We are now going to prove this claim. Every element of $Q_{\text {sat }}=K \cap \mathbb{Z}^{d}$ is a non-negative integral combination of elements of $B$. However $2 \boldsymbol{b}, \boldsymbol{b} \in B$, are already contained in $A$. Therefore $\boldsymbol{x} \in Q(A)$ is a saturation point if for every subset $\tilde{B}$ of $B$

$$
\boldsymbol{x}+\sum_{\boldsymbol{b} \in \tilde{B}} b \in Q .
$$

Now by (5)

$$
2 C \boldsymbol{b}_{F_{i}}^{*}+\sum_{\boldsymbol{b} \in \tilde{B}}\left(\left(2 \boldsymbol{b}_{F_{i}}^{*}+\boldsymbol{b}\right)-2 \boldsymbol{b}_{F_{i}}^{*}\right)=2(C-|\tilde{B}|) \boldsymbol{b}_{F_{i}}^{*}+\sum_{\boldsymbol{b} \in \tilde{B}}\left(2 \boldsymbol{b}_{F_{i}}^{*}+\boldsymbol{b}\right) \in Q .
$$

Therefore $2 C \boldsymbol{b}_{F_{i}}^{*}$ is a saturation point. This proves the theorem.

\section{Examples}

In this section we will go through some theorems with an example from Example 2.4 and with a defining matrix for $2 \times 2 \times 2 \times 2$ tables with three 2-marginals and a 3-marginal as the simplicial complex on 4 nodes [12][13][14][234] with levels of 2 on each node. 
5.1. $2 \times 4$ matrix from Example 2.4. Let $A$ be an integral matrix such that

$$
A=\left(\begin{array}{llll}
1 & 1 & 1 & 1 \\
0 & 2 & 3 & 4
\end{array}\right) .
$$

The cone $K$ is defined by $K=\operatorname{cone}(A)=\left\{\left(x_{1}, x_{2}\right)^{t}:-4 x_{1}+x_{2} \leq 0, x_{1}, x_{2} \in \mathbb{R}_{+}\right\}$. One-dimensional faces are $F_{1}=\left\{\left(x_{1}, 0\right)^{t}: x_{1} \in \mathbb{R}_{+}\right\}$and $F_{2}=\left\{\left(x_{1}, x_{2}\right)^{t}:-4 x_{1}+\right.$ $\left.x_{2}=0, x_{1}, x_{2} \in \mathbb{R}_{+}\right\}$. The set of holes $H$ consists of elements $\{(k, 1): k \in \mathbb{Z}, k \geq 1\}$ and $H_{0}$ consists of only one element $(1,1)^{t}$ since $Q_{\text {sat }} \cap\left((k, 1)^{t}-Q\right)=\left\{(i, 1)^{t}: i \in\right.$ $\mathbb{Z}, 1 \leq i \leq k\}$. Let $\boldsymbol{y}=(1,1)^{t}$. Also let $\boldsymbol{a}_{1}=(1,0)^{t}, \boldsymbol{a}_{2}=(1,2)^{t}, \boldsymbol{a}_{2}=(1,3)^{t}, \boldsymbol{a}_{2}=$ $(1,4)^{t}$.

Consider $\boldsymbol{y}+Q_{F_{1}}$. Note that $Q_{F_{1}}=\{(x, 0): x \in \mathbb{N}\}$ so $\boldsymbol{y}+Q_{F_{1}}=\{(x, 1)$ : $x \in \mathbb{N}, a \geq 1\}$. Note that $\boldsymbol{y}+Q_{F_{1}} \subset H$. Thus, by Proposition $\left[3.2, \boldsymbol{y}+Q_{F_{1}} \subset H\right.$ implies $F_{1}$ is nowhere saturated.

Now we examine whether $F_{1}$ and $F_{2}$ are nowhere saturated or almost saturated via Theorem 3.3. First, we will decide whether $F_{1}$ is nowhere saturated or almost saturated. Note that $\boldsymbol{a}_{1} \in F_{1}$, so we set the system of linear equations and inequalities such that:

(6)

$\boldsymbol{y}=x_{1} \boldsymbol{a}_{1}+x_{2} \boldsymbol{a}_{2}+x_{3} \boldsymbol{a}_{3}+x_{4} \boldsymbol{a}_{4}, x_{i} \in \mathbb{Z}$, for $i=1, \cdots, 4, x_{j} \geq 0$, for $j=2,3,4$.

We count the number of integral solutions in the system (6) via LattE (DeLoera et al. 2003]) and find out that there is no integral solution in the system. Thus, by Theorem 3.3. we know that $F_{1}$ is nowhere saturated.

Secondly, we will decide whether $F_{2}$ is nowhere saturated or almost saturated. Note that $\boldsymbol{a}_{4} \in F_{2}$, so we set the system of linear equations and inequalities such that:

$\boldsymbol{y}=x_{1} \boldsymbol{a}_{1}+x_{2} \boldsymbol{a}_{2}+x_{3} \boldsymbol{a}_{3}+x_{4} \boldsymbol{a}_{4}, x_{i} \in \mathbb{Z}$, for $i=1, \cdots, 4, x_{j} \geq 0$, for $j=1,2,3$.

We count the number of integral solutions in the system (7) via LattE and find out that there are two integral solutions in the system, namely $\left(x_{1}, x_{2}, x_{3}, x_{4}\right)=$ $(0,0,3,-2)$ and $\left(x_{1}, x_{2}, x_{3}, x_{4}\right)=(0,1,1,-1)$. Thus, by Theorem 3.3, we know that $F_{2}$ is almost saturated.

Now we are going to decide whether $F_{1}$ and $F_{2}$ are nowhere saturated or almost saturated via Theorem 3.4. Note that the Hilbert basis $B$ of the cone $K$ consists of 5 elements

$$
B=\left\{\boldsymbol{b}_{1}=(1,0)^{t}, \boldsymbol{b}_{2}=(1,1)^{t}, \boldsymbol{b}_{3}=(1,2)^{t}, \boldsymbol{b}_{4}=(1,3)^{t}, \boldsymbol{b}_{5}=(1,4)^{t}\right\} .
$$

Firstly for $F_{1}$, notice that $\boldsymbol{y}=\boldsymbol{b}_{2}$. Thus since the system (6) does not have an integral solution so $F_{1}$ is nowhere saturated by Theorem 3.4.

For $F_{2}$ we set the five systems of equations and inequalities such that:

$$
\begin{aligned}
\boldsymbol{b}_{k}=x_{1} \boldsymbol{a}_{1}+x_{2} \boldsymbol{a}_{2}+x_{3} \boldsymbol{a}_{3}+x_{4} \boldsymbol{a}_{4}, \\
x_{i} \in \mathbb{Z}, \text { for } i=1, \cdots, 4, \quad x_{j} \geq 0, \text { for } j=1,2,3, \text { for } k=1, \cdots, 5 .
\end{aligned}
$$

Using LattE we find out that all systems contain integral solutions, thus by Theorem 3.4. $F_{2}$ is almost saturated.

We are going to decide whether $F_{1}$ is nowhere saturated using Theorem 3.5 From Example 2.4 we know that $\min (S ; Q)=\left\{(1,2)^{t},(1,3)^{t},(1,4)^{t}\right\}$. We take $(1,2)^{t} \in \min (S ; Q)$. Then we notice that $(1,2)^{t}+Q_{F_{1}}$ is contained in $\min (S ; S)$, thus, by Theorem 3.5, $F_{1}$ is nowhere saturated. 
5.2. $2 \times 2 \times 2 \times 2$ tables with 2 -marginals and a 3 -marginal. Now we consider $2 \times 2 \times 2 \times 2$ tables with three 2 -marginals and a 3 -marginal as the simplicial complex on 4 nodes [12][13][14][234] with levels of 2 on each node. Takemura and Yoshida 2006] showed that the cardinality of $H$ for the semigroup defined by their matrix is infinite. Thus, we should like to investigate which face of the polyhedral cone defined by this matrix is almost saturated or nowhere saturated by Theorem 3.4 . To compute minimal Hilbert bases of cones, we used normaliz (Bruns and Koch 2001]) and to compute each hyperplane representation and vertex representation we used CDD (Fukuda 2005]) and Irs (Avis 2005]). Also we used 4ti2 (Hemmecke et al. 2005]) to compute defining matrices. To count the number of integral solutions in each system, we used LattE (DeLoera et al. [2003]).

After removing redundant rows (we removed redundant rows using cddlib) (Fukuda 2005]), $2 \times 2 \times 2 \times 2$ tables with 2-marginals and a 3 -marginal have a $12 \times 16$ defining matrix. Thus the semigroup is generated by 16 (column) vectors in $\mathbb{Z}^{12}$ such that:

10000010000100010001000

$0100 \begin{array}{lllllllllllllllll}0 & 1 & 0 & 0 & 0 & 1 & 0 & 0 & 0 & 1 & 0 & 0 & 0 & 1 & 0 & 0\end{array}$

$0 \begin{array}{llllllllllllllll}0 & 0 & 1 & 0 & 0 & 0 & 1 & 0 & 0 & 0 & 1 & 0 & 0 & 0 & 1 & 0\end{array}$

$0 \begin{array}{llllllllllllllllll}0 & 0 & 0 & 1 & 0 & 0 & 0 & 1 & 0 & 0 & 0 & 1 & 0 & 0 & 0 & 1\end{array}$

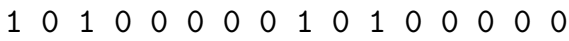

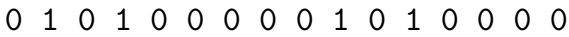

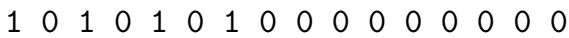

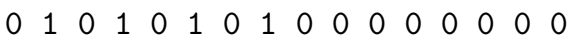

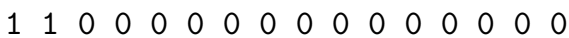

$0 \begin{array}{llllllllllllllll}0 & 0 & 1 & 1 & 0 & 0 & 0 & 0 & 0 & 0 & 0 & 0 & 0 & 0 & 0 & 0\end{array}$

$0 \begin{array}{llllllllllllllll}0 & 0 & 0 & 0 & 1 & 1 & 0 & 0 & 0 & 0 & 0 & 0 & 0 & 0 & 0 & 0\end{array}$

$0 \begin{array}{llllllllllllllll}0 & 0 & 0 & 0 & 0 & 0 & 0 & 0 & 1 & 1 & 0 & 0 & 0 & 0 & 0 & 0\end{array}$

All of these vectors are extreme rays of the cone (we verified via cddlib). The Hilbert basis of the cone generated by these 16 vectors consists of these 16 vectors and two additional vectors

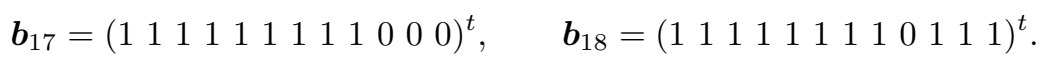

Using CDD we computed the system of 48 linear inequalities for defining the cone. Thus, the cone $K$ has 48 facets. The results of our experiments are in Table 1. To enumerate all faces, we used allfaces_gmp from cddlib.

From Table 1 we see that 64 almost saturated 2 dimensional faces are minimal. We have checked that all almost saturated faces with dimensions 3 or larger contain at least one of 64 almost saturated 2 dimensional faces. This implies that 2 nowhere saturated 8 dimensional faces are the maximal nowhere saturated faces. Therefore the most important faces to investigate are 2 nowhere saturated 8 dimensional faces and 64 almost saturated 2 dimensional faces. We give detailed descriptions of these faces.

For 2 nowhere saturated 8 dimensional faces, the set of extreme rays of a cone is the set of 8 columns of 16 generators for the semigroup, namely columns $1,2,7,8,11,12,13,14$. Since this 8 dimensional face is spanned by 8 vectors, it is a simplicial face. The extreme rays of the other cone is just the complement of the extreme rays of this cone. These two nowhere saturated faces are corresponding to [234] marginals. In order to see a picture, we let $x_{i}$ correspond to the $i$ th column 


\begin{tabular}{|c|c|c|c|}
\hline Dimension & \# of faces & \# of nowhere & \# of almost \\
\hline 11 & 48 & 0 & 48 \\
\hline 10 & 492 & 0 & 492 \\
\hline 9 & 2104 & 0 & 2104 \\
\hline 8 & 4898 & 2 & 4896 \\
\hline 7 & 6956 & 16 & 6940 \\
\hline 6 & 6440 & 56 & 6384 \\
\hline 5 & 4064 & 112 & 3952 \\
\hline 4 & 1796 & 140 & 1656 \\
\hline 3 & 560 & 112 & 448 \\
\hline 2 & 120 & 56 & 64 \\
\hline 1 & 16 & 16 & 0 \\
\hline
\end{tabular}

TABLE 1 . Faces for $2 \times 2 \times 2 \times 2$ tables with three 2 -marginals and a 3-marginal. The first column represents the dimension of faces, the second column represents the number of faces, the third column represents the number of nowhere saturated faces, and the fourth column represents the number of almost saturated faces.

of the defining matrix $A$ above. Let $T=\left(T_{i j k l}\right)$ be a $2 \times 2 \times 2 \times 2$ table where $i, j, k, l=1,2$. Then we can write 16 cells of a $2 \times 2 \times 2 \times 2$ table as Figure 4 . There are eight [234] marginals, i.e.

$$
\begin{aligned}
x_{1}+x_{2}=T_{+111}, & x_{3}+x_{4}=T_{+121}, \\
x_{5}+x_{6}=T_{+211}, & x_{7}+x_{8}=T_{+221}, \\
x_{9}+x_{10}=T_{+112}, & x_{11}+x_{12}=T_{+122}, \\
x_{13}+x_{14}=T_{+212}, & x_{15}+x_{16}=T_{+222} .
\end{aligned}
$$

Four marginals $\left\{T_{+111}, T_{+221}, T_{+122}, T_{+212}\right\}$ correspond to one face and and the other four marginals correspond to another face.

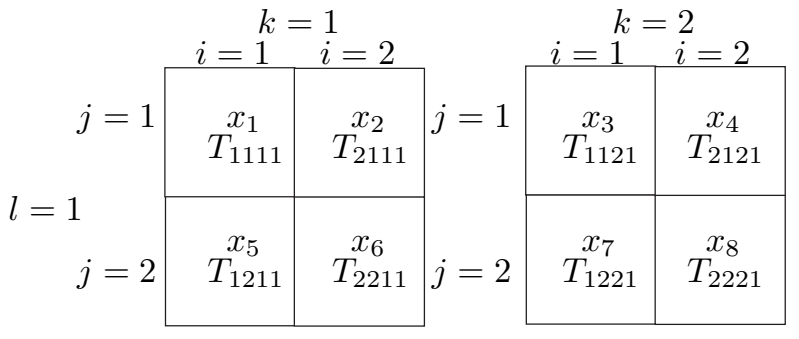

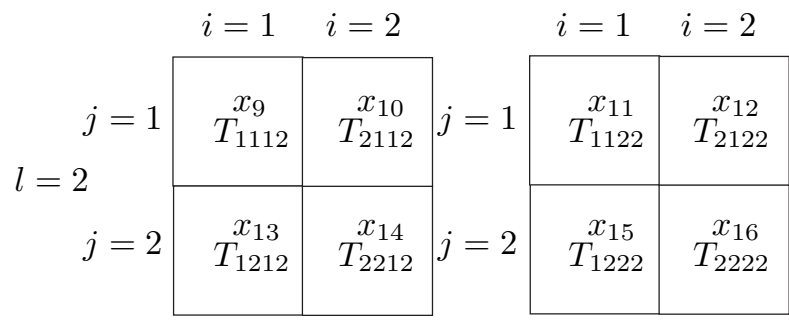

Figure $4.2 \times 2 \times 2 \times 2$ tables 
We now consider 64 almost saturated 2 dimensional faces. Each 2 dimensional face is spanned by two extreme rays and each extreme ray corresponds to a cell $T_{i j k l}$. Therefore each face can be identified with a pair of cells. Rather than listing all 64 faces, it is more instructive to consider symmetry of the problem and list only different types of the faces. Note that the semigroup has the symmetry with respect to interchanging the values $1 \leftrightarrow 2$ for each $i, j, k, l$ independently, and with respect to the permutation of indices $j, k, l$. Therefore the product group $S_{2} \times S_{2} \times S_{2} \times S_{2} \times S_{3}$ is naturally acting on the semigroup. By this action an almost saturated face is mapped to another almost saturated face. Detailed investigation of group invariance for Markov bases is given by Aoki and Takemura [2003] and Aoki and Takemura 2005. The orbits for 2 dimensional faces can be summarized as follows. The first index $i$ can not be interchanged with other $j$ or $k$ or $l$. So we first look at $i$. Then there are seven possible cases:

(1) Whether we have a common value of $i$ or different values of $i$ in the two rays.

(2) For each case, we can look at the number of common values of $j, k, l$ in the two rays.

So there are seven possible cases as following:

(1) $i$ is common and

(a) $j, k, l$ are all different.

(b) one of $j, k, l$ is common.

(c) two of $j, k, l$ are common.

(2) $i$ is different and

(a) $j, k, l$ are all different.

(b) one of $j, k, l$ is common.

(c) two of $j, k, l$ are common.

(d) $j, k, l$ are all common.

We counted the number of almost saturated 2 dimensional faces for each type among the seven cases and the results are the following:

- There are 8 Type 1a almost saturated 2 dimensional faces.

- There are 24 Type $1 \mathrm{c}$ almost saturated 2 dimensional faces.

- There are 8 Type 2 a almost saturated 2 dimensional faces.

- There are 24 Type $2 \mathrm{C}$ almost saturated 2 dimensional faces.

There are no almost saturated 2 dimensional faces of other types. In order to make this classification based on symmetry clear, we give the following example.

Example 5.1. The cone generated by the 10 th and the 13 th columns of the matrix and the cone generated by the 6th and the 13th columns of the matrix are two dimensional almost saturated faces of the cone among 64 cones. Since the 6th column represents $T_{2121}$, the 10 th column represents $T_{2112}$, and the 13 th column represents $T_{1122}$, we have the index set for the cones:

\begin{tabular}{|l|c|c|}
\hline & 1st cone & 2nd cone \\
\hline 1st ray & 1122 & 1122 \\
2nd ray & 2112 & 2121 \\
\hline
\end{tabular}

Note that for both cones, the value of $i$ in each ray has different value of the other. Thus we have Case 2. Now we look at other indices. $j$ is 1 in both cones. However, $k$ has different values 1 and 2 in the first cone but $k$ has the same value in the second 
cone. $l$ has different values 1 and 2 in the second cone but $l$ has the same value in the first cone. Thus the number of indices having the same value is two. Therefore, the two cones are the same type, namely Type 20. In fact just by exchanging $k \leftrightarrow l$, the first cone is mapped to the second cone in this example.

\section{Acknowledgment}

The authors would like to thank the referees for giving us helpful comments to improve this paper.

\section{References}

K. Aardal and A. K. Lenstra. Hard equality constrained integer knapsacks. In Integer programming and combinatorial optimization, volume 2337 of Lecture Notes in Comput. Sci., pages 350-366. Springer, Berlin, 2002.

K. Aardal, C. A. J. Hurkens, and A. K. Lenstra. Solving a system of linear Diophantine equations with lower and upper bounds on the variables. Math. Oper. Res., 25(3):427-442, 2000. ISSN 0364-765X.

K. Aardal, R. Weismantel, and L. A. Wolsey. Non-standard approaches to integer programming. Discrete Appl. Math., 123(1-3):5-74, 2002. ISSN 0166-218X. Workshop on Discrete Optimization, DO'99 (Piscataway, NJ).

S. Aoki and A. Takemura. Invariant minimal markov basis for sampling contingency tables with fixed marginals. Technical Report METR 2003-25, University of Tokyo, 2003.

S. Aoki and A. Takemura. The largest group of invariance for markov bases and toric ideals. Technical Report METR 2005-14, University of Tokyo, 2005.

D. Avis. Irs and lrslib, $2005 . \quad$ Available via http://cgm.cs.mcgill.ca/ avis/C/lrslib/.

A. Barvinok and K. Woods. Short rational generating functions for lattice point problems. Journal of the American Mathematical Society, 16:957-979, 2003.

W. Bruns and J. Gubeladze. Unimodular covers of multiples of polytopes. Documenta Math, 7:463-480, 2002a.

W. Bruns and R. Koch. NORMALIZ, computing normalizations of affine semigroups, 2001. Available via anonymous ftp from ftp//ftp.mathematik.uni-onabrueck.de/pub/osm/kommalg/software/.

W. Bruns, J. Gubeladze, and N. Trung. Normal polytopes, triangulations and koszul algebras. J. Reine Angew. Math., 485:123-160, 1997.

Winfried Bruns and Joseph Gubeladze. Semigroup rings and discrete geometry. Séminaires et Congrès, 6:43-127, 2002b.

L. H. Cox. On properties of multi-dimensional statistical tables. Preprint, 2000.

L. H. Cox. Bounds on entries in 3-dimensional contingency tables subject to given marginal totals. In Inference Control in Statistical Databases, pages 21-33, 2002.

J. A. DeLoera, D. Haws, R. Hemmecke, P. Huggins, J. Tauzer, and R. Yoshida. A user's guide for latte v1.1, 2003. software package LattE is available at http://www. math.ucdavis.edu/ latte/.

A. Dobra, A. F. Karr, and A. P. Sanil. Preserving confidentiality of highdimensional tabulated data: statistical and computational issues. Stat. Comput., 13(4):363-370, 2003. ISSN 0960-3174.

K. Fukuda. cdd and cdd+, the cdd and cdd plus, 2005. Available via http://www.cs.mcgill.ca/ fukuda/soft/cdd_home/cdd.html. 
I. M. Gelfand, M. M. Kapranov, and A. V. Zelevinsky. Discriminants, Resultants, and Multidimensional Determinants. Birkhäuser, 1994.

R. Hemmecke, R. Hemmecke, and P. Malkin. 4ti2 version 1.2-computation of Hilbert bases, Graver bases, toric Gröbner bases, and more. Available at www.4ti2.de, sep. 2005.

J.M. Kantor and K. S. Sarkaria. On primitive subdivisions of an elementary tetrahedron, 2001 URL http://www. ihes.fr/PREPRINTS/M01/Resu/resu-M01-23.html.

E. Miller and B. Sturmfels. Combinatorial Commutative Algebra. Springer-Verlag, New York, 2005. ISBN 0-3872-2356-8.

H. Ohsugi and T. Hibi. Toric ideals arising from contingency tables. 2006. Proceedings of the Ramanujan Mathematical Society's Lecture Notes Series, to appear.

R. P. Stanley. Enumerative combinatorics. Vol. 1, volume 49 of Cambridge Studies in Advanced Mathematics. Cambridge University Press, Cambridge, 1997. ISBN 0-521-55309-1; 0-521-66351-2. With a foreword by Gian-Carlo Rota, Corrected reprint of the 1986 original.

B. Sturmfels. Grobner Bases and Convex Polytopes. Univ. Lectures Series, No 8. American Mathematical Society, Rhode Island, 1996. ISBN 0-8218-0487-1.

A. Takemura and R. Yoshida. A generalization of the integer linear infeasibility problem, 2006. URL http://arXiv.org/abs/math.ST/0603108.

M. Vlach. Conditions for the existence of solutions of the three-dimensional planar transportation problem. Discrete Appl. Math., 13(1):61-78, 1986. ISSN 0166$218 \mathrm{X}$.

Department of Mathematical Informatics, University of Tokyo, Bunkyo, Tokyo, JAPAN.TAKEMURA@STAT.T.U-TOKYO.AC.JP

Department of Statistics, University of Kentucky, Lexington, KY USA. RuRiko@ms.uky.edu 